\title{
AN UPPER BOUND ON THE DIAMETER OF A GRAPH FROM EIGENVALUES ASSOCIATED WITH ITS LAPLACIAN*
}

\author{
F. R. K. CHUNG ${ }^{\dagger}$, V. FABER ${ }^{\ddagger}$, AND THOMAS A. MANTEUFFEL $\ddagger$
}

Abstract. The authors give a new upper bound for the diameter $D(G)$ of a graph $G$ in terms of the eigenvalues of the Laplacian of $G$. The bound is

$$
D(G) \leq\left\lfloor\frac{\cosh ^{-1}(n-1)}{\cosh ^{-1}\left(\frac{\lambda_{n}+\lambda_{2}}{\lambda_{n}-\lambda_{2}}\right)}\right\rfloor+1,
$$

where $0 \leq \lambda_{2} \leq \cdots \leq \lambda_{n}$ are the eigenvalues of the Laplacian of $G$ and where \lfloor\rfloor is the floor function.

Key words. Laplacian, diameter, eigenvalues

AMS subject classification. $05 \mathrm{C}$

1. Introduction. Suppose that $G$ is a connected graph (undirected or directed). For two vertices $u$ and $v$ in $G$, the distance between $u$ and $v$, denoted by $d(u, v)$, is the length of a shortest path joining $u$ and $v$ in $G$. The diameter of $G$, denoted by $D(G)$, is the maximum distance over all pairs of vertices in $G$. The diameter is one of the graph variants that are not only of theoretical interest but also have many practical applications. In many communication models, diameter plays a key role in performance and cost optimization in network design when the time delay or signal degradation is proportional to the number of links that a message must travel. Numerous applications include circuit design, data representation, and parallel and distributive computing.

Let $A$ denote the adjacency matrix of $G$. That is, $(A)_{i j}=1$ if there is an edge between $i$ and $j$ (or from $i$ to $j$ ), and it is 0 otherwise. Let $d_{i}$ denote the degree of the $i$ th vertex. When all $d_{i}$ 's are equal to $k$, we say that $G$ is $k$-regular. The Laplacian of $G$ is defined to be the matrix $Q=Q(G)$, where $(Q)_{i i}=d_{i}$ and $(Q)_{i j}=-A_{i j}$ if $i \neq j$. When a directed graph has the property that the in-degree is equal to the outdegree at every vertex, we can define the Laplacian analogously. The smallest nonzero eigenvalue, denoted by $\lambda(G)$, of $Q(G)$ can be used to derive various properties of $G$. It was shown in [12], [13] that $\lambda(G)$ provides useful bounds for the expanding properties of $G$. Using the expanding properties, Alon and Milman [1] deduced the following upper bound for the diameter for graphs with maximum degree $k: D(G) \leq 2 \sqrt{2 k / \lambda} \log _{2} n$.

This bound was later improved [3] to $D(G) \leq\lceil\log (n-1) / \log (k / \bar{\lambda})\rceil$, where $\bar{\lambda}$ denotes the second largest eigenvalue (in absolute value) of $A$. In this paper, we show that $D(G) \leq\left\lfloor\cosh ^{-1}(n-1) / \cosh ^{-1}(k / \bar{\lambda})\right\rfloor+1$, which is a special case of the following bound for general graphs:

$$
D(G) \leq\left\lfloor\frac{\cosh ^{-1}(n-1)}{\cosh ^{-1}\left(\frac{\lambda_{n}+\lambda_{2}}{\lambda_{n}-\lambda_{2}}\right)}\right\rfloor+1,
$$

\footnotetext{
* Received by the editors June 19, 1991; accepted for publication (in revised form) March $23,1993$.

† Bell Communications Research, Morristown, New Jersey 07960.

$\ddagger$ Los Alamos National Laboratory, Los Alamos, New Mexico 87545.
} 
where $0 \leq \lambda_{2} \leq \cdots \leq \lambda_{n}$ denote the eigenvalues of the Laplacian of $G$.

Some diameter bounds for directed graphs are also derived. Although these bounds are not as good as those for undirected cases, the techniques can be useful for bounding the diameter for Cayley graphs or on the factorization of groups. Namely, for a group $H$ and a set $S$ of generators, it is often desirable to find the least integer $m=D_{S}(H)$ such that every element of $H$ can be written as the product of no more than $m$ elements in $S$ (repetitions are allowed). The upper bounds for diameters can then be applied for bounding $D_{S}(H)$ when the graph is considered to be the Cayley graph on $H$ determined by $S$.

2. Diameter of a graph. Let $G$ be a directed graph and $A$ its adjacency matrix. Since the $i, j$ entry of $A^{k}$ is the number of directed paths from vertex $i$ to vertex $j$ of length $k$, the diameter $D$ is the smallest natural number such that, given any $i$ and $j$, one of the matrices in the set $\left\{A^{m} \mid 0 \leq m \leq D\right\}$ has a nonzero $i, j$ entry. If a new matrix $\bar{A}=A-\Lambda$ is formed from $A$ by subtracting any diagonal matrix $\Lambda$, the powers of $\bar{A}$ still have the same significance as the powers of $A$. To see this, note that $\bar{A}^{m}$ has a nonzero $i, j$ entry only if there is a directed path of length at most $m$ in $G$ from vertex $i$ to vertex $j$; if the distance from vertex $i$ to vertex $j$ in $G$ is $m$, then $\bar{A}^{m}$ has a nonzero $i, j$ entry. Thus, the diameter $D$ is the smallest natural number such that, given any $i \neq j$, one of the matrices in the set $\left\{\bar{A}^{m} \mid 0 \leq m \leq D\right\}$ has a nonzero $i, j$ entry.

We can now introduce polynomials into the definition of diameter. The statement that the $i, j$ entry of at least one of the matrices in the set $\left\{\bar{A}^{m} \mid 0 \leq m \leq D\right\}$ is nonzero is equivalent to the statement that there exists a polynomial $p_{m}$ of degree $m$ less than or equal to $D$ such that the $i, j$ entry of $p_{m}(\bar{A})$ is nonzero. Thus the diameter $D$ is the smallest natural number such that, given any $i \neq j$, there exists a polynomial $p_{m}$ of degree $m \leq D$ such that the $i, j$ entry of $p_{m}(\bar{A})$ is nonzero.

In fact, it can be easily seen that $D$ is equal to the smallest natural number $m$ for which there exists a polynomial $p_{m}$ of degree $m$ such that all off-diagonal entries of $p_{m}(\bar{A})$ are nonzero.

3. A fundamental inequality involving the Laplacian. In this section, it is essential to assume that the in-degree of each vertex of $G$ is equal to its out-degree. We denote by $d_{i}$ this common value for the vertex $i$. The Laplacian of $G$ is defined to be $Q=\operatorname{diag}\left(d_{i}\right)-A$. Note that $Q u_{1}=Q^{*} u_{1}=0$, where $u_{1}=(1 / \sqrt{n})(1,1, \ldots, 1)^{*}$; so $Q J=J Q=0$, where $J=n u_{1} u_{1}^{*}$ is the matrix all of whose entries are 1 . Let $e_{i}$ be the unit vector in the $i$ th direction.

Lemma 3.1. Let $B$ be an $n \times n$ matrix with the properties $B u_{1}=B^{*} u_{1}=0$. Then

$$
\left|B_{r, s}\right| \leq\|B\|\left(1-\frac{1}{n}\right) .
$$

Proof. Define $f_{i}=e_{i}-(1 / \sqrt{n}) u_{1}$. Then $\left(f_{r}, u_{1}\right)=0,\left(u_{1}, B f_{r}\right)=0$, and $\left\|f_{r}\right\|=$ $\sqrt{1-1 / n}$. Thus

$$
\left|B_{r, s}\right|=\left|\left(e_{r}, B e_{s}\right)\right|=\left|\left(f_{r}, B f_{s}\right)\right| \leq\left\|f_{r}\right\|\|B\|\left\|f_{s}\right\|=\|B\|\left(1-\frac{1}{n}\right) .
$$

This proves the lemma.

THEOREM 3.2. Let $p_{m}(x)$ be a polynomial of degree $m$ with $p_{m}(0)=1$ such that

$$
\left\|p_{m}(Q)-\frac{J}{n}\right\|<\frac{1}{n-1}
$$


Then $D \leq m$.

Proof. Let $B=p_{m}(Q)-J / n$. We have

$$
B u_{1}=p_{m}(Q) u_{1}-\frac{J}{n} u_{1}=p_{m}(0) I u_{1}-u_{1}=0
$$

and similarly $B^{*} u_{1}=0$. Thus Lemma 3.1 applies, and

$$
\begin{aligned}
\left(p_{m}(Q)\right)_{r, s} & \geq\left(\frac{J}{n}\right)_{r, s}-\left|B_{r, s}\right| \\
& \geq \frac{1}{n}-\|B\|\left(1-\frac{1}{n}\right) \\
& >\frac{1}{n}-\frac{1}{n-1}\left(1-\frac{1}{n}\right) \\
& =0 .
\end{aligned}
$$

Thus, by our earlier remarks on diameter, $D \leq m$. This proves the theorem.

4. The Chebychev polynomials. The Chebychev polynomials of the first kind are given by

$$
\begin{gathered}
T_{0}(z)=1 \\
T_{1}(z)=z \\
T_{n+1}(z)=2 z T_{n}(z)-T_{n-1}(z), \quad n \geq 1 .
\end{gathered}
$$

They may also be written as

$$
T_{n}(z)=\cosh \left(n \cosh ^{-1}(z)\right) .
$$

The Chebychev polynomials have the following interesting optimality property [14]. Let $S_{n}$ be the set of all polynomials, $s_{n}(x)$, of degree $n$ such that $s_{n}(0)=1$. Let $[a, b]$ be an interval on the real line to the right of the origin. Then there exists a unique $t_{n} \in S_{n}$ such that

$$
\max _{x \in[a, b]}\left|t_{n}(x)\right|=\min _{s_{n} \in S_{n}} \max _{x \in[a, b]}\left|s_{n}(x)\right|
$$

and

$$
t_{n}(x)=\frac{T_{n}\left(\frac{a+b-2 x}{b-a}\right)}{T_{n}\left(\frac{a+b}{b-a}\right)}
$$

Furthermore,

$$
\max _{x \in[a, b]}\left|t_{n}(x)\right|=t_{n}(a)=\frac{1}{T_{n}\left(\frac{a+b}{b-a}\right)} .
$$

This result can be extended to a class of closed ellipses in the complex plane not containing the origin with foci lying on the real line or foci that are a complex conjugate pair. In particular, for $\delta>0$ and $\gamma>0$ real, if the ellipse $E$ has center $\delta$, foci $\delta \pm \gamma$, and semi-major axis of length $\alpha$ with $\gamma \leq \alpha<\delta$, then

$$
t_{n}(z)=\frac{T_{n}\left(\frac{\delta-z}{\gamma}\right)}{T_{n}\left(\frac{\delta}{\gamma}\right)}
$$


has the property

$$
\max _{z \in E}\left|t_{n}(z)\right|=\frac{T_{n}\left(\frac{\alpha}{\gamma}\right)}{T_{n}\left(\frac{\delta}{\gamma}\right)} .
$$

Furthermore, if $\gamma \leq \alpha \leq \alpha_{n}^{*}<\delta$, then

$$
\max _{z \in E}\left|t_{n}(z)\right|=\min _{s_{n} \in S_{n}} \max _{z \in E}\left|s_{n}(z)\right|
$$

Here $\alpha_{n}^{*}$ is close to $\delta$ and depends on $n$ in a complicated way. However, $\lim _{n \rightarrow \infty} \alpha_{n}^{*}=\delta$, and thus the Chebychev polynomials are asymptotically optimal [10], [6], [7]. If, on the other hand, the ellipse $E$ has center $\delta$, foci $\delta \pm i \gamma$, and semimajor axis of length $\alpha, \gamma \leq \alpha<\left(\delta^{2}+\gamma^{2}\right)^{1 / 2}$, then

$$
t_{n}(z)=\frac{T_{n}\left(\frac{\delta-z}{i \gamma}\right)}{T_{n}\left(\frac{\delta}{i \gamma}\right)}
$$

has real coefficients and for $n$ even satisfies

$$
\frac{T_{n}\left(\frac{\alpha}{\gamma}\right)}{T_{n}\left[\left(1+\left(\frac{\delta}{\gamma}\right)^{2}\right)^{1 / 2}\right]}=\max _{z \in Z}\left|t_{n}(z)\right|
$$

Again, if $\gamma<\alpha \leq \alpha_{n}^{*}<\left(\delta^{2}+\gamma^{2}\right)^{1 / 2}$, then

$$
\max _{z \in E} t_{n}(z)=\min _{s_{n} \in S_{n}} \max _{z \in E}\left|s_{n}(z)\right|
$$

where $\alpha_{n}^{*}$ is close to $\left(\delta^{2}+\gamma^{2}\right)^{1 / 2}$ and $\lim _{n \rightarrow \infty} \alpha_{n}^{*}=\left(\delta^{2}+\gamma^{2}\right)^{1 / 2}[6],[7]$. For $n$ odd and $\gamma \leq \alpha<\left(\delta^{2}+\gamma^{2}\right)^{1 / 2}$, we have

$$
\frac{T_{n}\left(\frac{\alpha}{\gamma}\right)}{\left[T_{n}^{2}\left(\left(1+\left(\frac{\delta}{\gamma}\right)^{2}\right)^{1 / 2}\right)-1\right]^{1 / 2}}=\max _{z \in E}\left|t_{n}(z)\right| \leq \frac{T_{n}\left(\frac{\alpha}{\gamma}\right)}{\left(T_{n}^{2}\left(\frac{\alpha}{\gamma}\right)-1\right)^{1 / 2}} \min _{s_{n} \in S_{n}} \max _{z \in E}\left|s_{n}(z)\right| .
$$

Note that for $\alpha>\gamma$, we have $\lim _{n \rightarrow \infty} T_{n}(\alpha / \gamma) /\left(T_{n}^{2}(\alpha / \gamma)-1\right)^{1 / 2}=1$, and thus the Chebychev polynomials are asymptotically optimal for $n$ odd as well. We only focus on real foci or even $n$.

Using the definition $T_{n}(z)=\cosh \left(n \cosh ^{-1}(z)\right)$ and the formula $\cosh ^{-1}(z)=$ $\ln \left(z+\left(z^{2}-1\right)^{1 / 2}\right)$, we may write

$$
g(z)=z+\sqrt{z^{2}-1}, \quad f(z)=z+\sqrt{z^{2}+1}
$$

then

$$
\frac{T_{n}\left(\frac{\alpha}{\gamma}\right)}{T_{n}\left(\frac{\delta}{\gamma}\right)}=\frac{g\left(\frac{\alpha}{\gamma}\right)^{n}+g\left(\frac{\alpha}{\gamma}\right)^{-n}}{g\left(\frac{\delta}{\gamma}\right)^{n}+g\left(\frac{\delta}{\gamma}\right)^{-n}}
$$


and

$$
\frac{T_{n}\left(\frac{\alpha}{\gamma}\right)}{T_{n}\left(\frac{\left(\delta^{2}+\gamma^{2}\right)^{1 / 2}}{\gamma}\right)}=\frac{g\left(\frac{\alpha}{\gamma}\right)^{n}+g\left(\frac{\alpha}{\gamma}\right)^{-n}}{f\left(\frac{\delta^{2}}{\gamma}\right)^{n}+f\left(\frac{\delta}{\gamma}\right)^{-n}} .
$$

Thus, once $\delta, \gamma$, and $\alpha$ are known, these bounds can be readily computed. These ideas have application to iterative methods for the solution of linear systems (see [10], [12]).

5. Bounding the diameter of an undirected graph from Chebychev polynomials. Let $Q$ denote the Laplacian matrix of an undirected graph on $n$ vertices. The following basic facts are summarized in [13].

Facts 5.1. Let $G$ be an undirected graph. Then

(a) $Q(G)$ has only real eigenvalues and a complete set of orthonormal eigenvectors,

(b) $Q(G)$ is positive semidefinite,

(c) its smallest eigenvalue is $\lambda_{1}=0$, and a corresponding eigenvector is $u_{1}=$ $(1 / \sqrt{n})(1,1, \ldots, 1)^{*}$. The multiplicity of 0 as an eigenvalue is equal to the number of components of $G$.

We assume that the eigenvalues of $Q(G)$ are ordered $\lambda_{1} \leq \lambda_{2} \leq \cdots \leq \lambda_{n}$ in increasing order and repeated according to their multiplicity. Thus $\lambda_{1}=0$ and $\lambda_{2}>0$ if and only if $G$ is connected. The following facts are known.

Facts 5.2. Let $G$ have $n$ vertices. Then

(a) $\lambda_{2} \leq n /(n-1) \min \{d(v) \mid v \in V(G)\}$,

(b) $\lambda_{n} \leq \max \{d(u)+d(v) \mid u v \in E(G)\}$, and, if $G$ is connected, equality holds if and only if $G$ is bipartite and semiregular.

(c) $\lambda_{n} \leq n$ with equality if and only if the complement of $G$ is not connected.

(d) $\sum_{i=1}^{n} \lambda_{i}=2|E(G)|=\sum_{v \in V} d(v)$,

(e) $\lambda_{n} \geq n /(n-1) \max \{d(v) \mid v \in V(G)\}$.

(f) $\lambda_{n} \geq \max \left\{\sqrt{(d(v)-d(u))^{2}+4} \mid u, v \in V(G), u \neq v\right\}$.

THEOREM 5.3. For an undirected graph $G$, we have

$$
D(G) \leq\left\lfloor\frac{\cosh ^{-1}(n-1)}{\cosh ^{-1} \frac{\lambda_{n}+\lambda_{2}}{\lambda_{n}-\lambda_{2}}}\right\rfloor+1 .
$$

Proof. Let

$$
m \leq\left\lfloor\frac{\cosh ^{-1}(n-1)}{\cosh ^{-1} \frac{\lambda_{n}+\lambda_{2}}{\lambda_{n}-\lambda_{2}}}\right]+1
$$

Then

$$
m>\frac{\cosh ^{-1}(n-1)}{\cosh ^{-1} \frac{\lambda_{n}+\lambda_{2}}{\lambda_{n}-\lambda_{2}}} .
$$

This can be rewritten as

$$
T_{m}\left(\frac{\lambda_{n}+\lambda_{2}}{\lambda_{n}-\lambda_{2}}\right)=\cosh \left(m \cosh ^{-1} \frac{\lambda_{n}+\lambda_{2}}{\lambda_{n}-\lambda_{2}}\right)>n-1 .
$$

We know by our discussion in $\S 4$ that if we let

$$
p_{m}(x)=\frac{T_{m}\left(\frac{\lambda_{n}+\lambda_{2}-2 x}{\lambda_{n}-\lambda_{2}}\right)}{T_{m}\left(\frac{\lambda_{n}+\lambda_{2}}{\lambda_{n}-\lambda_{2}}\right)}
$$


then $p_{m}(0)=1$, and

$$
\max _{2 \leq i \leq n}\left|p_{m}\left(\lambda_{i}\right)\right|=\frac{1}{T_{m}\left(\frac{\lambda_{n}+\lambda_{2}}{\lambda_{n}-\lambda_{2}}\right)}<\frac{1}{n-1} .
$$

Now since $Q$ is symmetric, we can write $Q$ as

$$
Q=\sum_{i=2}^{n} \lambda_{i} u_{i} u_{i}^{*}
$$

where the $u_{i}$ are orthonormal. Note that $u_{1}$ is as defined in $\S 3$ and that $\lambda_{1}=0$. Thus

$$
p_{m}(Q)-\frac{J}{n}=\sum_{i=2}^{n} p_{m}\left(\lambda_{i}\right) u_{i} u_{i}^{*}
$$

and

$$
\left\|p_{m}(Q)-\frac{J}{n}\right\|=\max _{2 \leq i \leq n}\left|p_{m}\left(\lambda_{i}\right)\right|<\frac{1}{n-1} .
$$

Thus, by Theorem 3.2 , we have $D \leq m$, which completes the proof.

Remark. Theorem 5.3 would be more asthetically pleasing if it yielded

$$
D(G) \leq\left\lceil\frac{\cosh ^{-1}(n-1)}{\cosh ^{-1}\left(\frac{\lambda_{n}+\lambda_{2}}{\lambda_{n}-\lambda_{2}}\right)}\right\rceil .
$$

This inequality fails only if $D(G)=m+1$ with

$$
m=\frac{\cosh ^{-1}(n-1)}{\cosh ^{-1}\left(\frac{\lambda_{n}+\lambda_{2}}{\lambda_{n}-\lambda_{2}}\right)} .
$$

Does such a graph $G$ exist? We easily see from the proof of Theorems 3.2 and 5.3 that for such a graph $G$, the Laplacian has eigenvector decomposition

$$
Q=\sum_{i} \lambda_{i} u_{i} u_{i}^{*}
$$

where $0=\lambda_{1}<\lambda_{2} \leq \lambda_{3} \cdots<\lambda_{n}$. There is a set of $m+1$ eigenvalues $\left\{\lambda_{k_{i}} \mid i=\right.$ $1, \ldots, m+1\}$ such that $\left|p_{m}\left(\lambda_{k_{i}}\right)\right|=1 /(n-1)$, where $p_{m}(\lambda)$ is the Chebychev polynomial defined in Theorem 5.3. That is,

$$
\lambda_{k_{i}}=\frac{\lambda_{n}+\lambda_{2}}{2}-\frac{\lambda_{n}-\lambda_{2}}{2} \cos \left(\frac{(i-1) \pi}{m}\right) \quad \text { for } i=1, \ldots, m+1 .
$$

Note that $\lambda_{2}=\lambda_{k_{1}}, \lambda_{n}=\lambda_{k_{m+1}}$. There is a unique pair of indices $r \neq s$ such that $\left\langle e_{r}, Q^{j} e_{s}\right\rangle=0$ for $i=0, \ldots, m$. Furthermore,

$$
\begin{aligned}
& e_{r}=\frac{1}{\sqrt{n}} u_{1}+\sum_{i=1}^{m+1} \alpha_{j} u_{k_{i}}, \\
& e_{s}=\frac{1}{\sqrt{n}} u_{1}+\sum_{i=1}^{m+1} \alpha_{i}(-1)^{i} u_{k_{i}},
\end{aligned}
$$


where

$$
\alpha_{i}^{2}=\frac{(-1)^{i-1}}{n} \frac{\prod_{j \neq i} \lambda_{k_{i}}}{\prod_{j \neq i}\left(\lambda_{k_{j}}-\lambda_{k_{i}}\right)} .
$$

Finally, we have, for $j=1, \ldots, m$,

$$
\sum_{i \text { odd }} \alpha_{i}^{2} \lambda_{k_{i}}^{j}=\sum_{i \text { even }} \alpha_{i}^{2} \lambda_{k_{i}}^{j}=\left\langle e_{r}, Q^{j}, e_{r}\right\rangle=\left\langle e_{s}, Q^{j} e_{s}\right\rangle
$$

which implies that $d(r)=d(s)$, while

$$
\sum_{i \text { odd }} \alpha_{i}^{2}=\frac{1}{2}, \quad \sum_{i \text { even }} \alpha_{i}^{2}=\frac{1}{2}-\frac{1}{n} .
$$

It is not known if graphs satisfying these conditions exist.

The following corollary is useful in conjunction with the estimates given in Facts 5.2 .

COROLLARY 5.4. Let $s_{2}$ and $s_{n}$ be estimates of $\lambda_{2}$ and $\lambda_{n}$, respectively, such that

$$
0<s_{2} \leq \lambda_{2} \leq \lambda_{n} \leq s_{n}
$$

Then

$$
D(G) \leq\left\lfloor\frac{\cosh -1(n-1)}{\cosh ^{-1} \frac{s_{n}+s_{2}}{s_{n}-s_{2}}}\right\rfloor+1 .
$$

Proof. It is easy to see that

$$
\cosh ^{-1} \frac{\lambda_{n}+\lambda_{2}}{\lambda_{n}-\lambda_{2}} \geq \cosh ^{-1} \frac{s_{n}+s_{2}}{s_{n}-s_{2}}
$$

COROllary 5.5. Let $A$ denote the adjacency matrix of a k-regular graph $G$. Suppose that $A$ has second largest eigenvalue $\tilde{\lambda}$ (in absolute value). Then

$$
D(G) \leq\left\lfloor\frac{\cosh ^{-1}(n-1)}{\cosh ^{-1}(k / \tilde{\lambda})}\right\rfloor+1 .
$$

Proof. It is easy to see that

$$
k-\tilde{\lambda} \leq \lambda_{2} \leq \lambda_{n} \leq k+\tilde{\lambda}
$$

Therefore

$$
D(G) \leq\left\lfloor\frac{\cosh ^{-1}(n-1)}{\cosh ^{-1} \frac{\lambda_{n}+\lambda_{2}}{\lambda_{n}-\lambda_{2}}}\right\rfloor+1 \leq\left\lfloor\frac{\cosh ^{-1}(n-1)}{\cosh ^{-1}(k / \tilde{\lambda})}\right\rfloor+1 .
$$

We remark that

$$
\frac{\cosh ^{-1}(n-1)}{\cosh ^{-1}(k / \tilde{\lambda})}<\frac{\ln (n-1)}{\ln (k / \tilde{\lambda})}
$$

except for the complete graph; thus Theorem 5.3 yields an improvement over the bound $D(G) \leq\lceil\ln (n-1) / \ln (k / \tilde{\lambda})\rceil$ in $[3]$. 
Remark. Chebychev polynomials have also been used to bound the diameter of Ramanujan graphs by Lubotzky, Phillips, and Sarnak [9] and for general graphs by Sarnak [16].

6. General directed graphs. In this section, we examine the estimates we can make for the diameter of general directed graphs $G$. Our starting point is Theorem 3.2 , and, as in that section, we assume that the in-degree of each vertex of $G$ equals its out-degree. (This holds if and only if $G$ is Eulerian.) Again, $Q(G)$ is the Laplacian of $G$.

THEOREM 6.1. If $\rho$ and $\lambda$ are such that $1 \geq \rho>\|I-\lambda Q-J / n\|$, then

$$
D(G) \leq\left\lceil\frac{\ln (n-1)}{\ln \frac{1}{\rho}}\right\rceil .
$$

Proof. Let

$$
m=\left\lceil\frac{\ln (n-1)}{\ln \left(\frac{1}{\rho}\right)}\right\rceil .
$$

So,

$$
m \geq \frac{\ln (n-1)}{\ln \left(\frac{1}{\rho}\right)}
$$

Let $p_{m}(x)=(1-\lambda x)^{m}$. Then

$$
p_{m}(Q)-\frac{J}{n}=\left(I-\lambda Q-\frac{J}{n}\right)^{m}
$$

so

$$
\begin{aligned}
\left\|p_{m}(Q)-\frac{J}{n}\right\| & =\left\|\left(I-\lambda Q-\frac{J}{n}\right)^{m}\right\| \\
& \leq\left\|I-\lambda Q-\frac{J}{n}\right\|^{m} \\
& <\rho^{m}=e^{-m \ln (1 / \rho)} \\
& \leq e^{-\ln (n-1)}=\frac{1}{n-1} .
\end{aligned}
$$

Thus, by Theorem $3.2, D \leq m$. This proves the theorem.

From this theorem, we see that it would be useful to know that

$$
\Phi=\min _{\lambda}\left\|I-\lambda Q-\frac{J}{n}\right\|
$$

We can give an estimate of $\Phi$ to show that this minimum is less than 1 when $G$ is connected. If $B$ is a matrix, we let $B_{S}=\left(B+B^{T}\right) / 2$ be the symmetric part of $B$ and we let $B_{A}=\left(B-B^{T}\right) / 2$ be the antisymmetric part of $B$. If $G$ is connected, then $Q_{S}$ is the Laplacian of a weighted connected undirected graph (see [13]), so $\sigma=\lambda_{2}\left(Q_{S}\right)>0$. We let $\mu=\|Q\|$. In the proof of Theorem 6.3 , we show that $\Phi \leq \sqrt{\mu^{2}-\sigma^{2}} / \mu$, so $\Phi<1$ when $G$ is connected. First, we need a technical lemma.

LEMMA 6.2. Let $\mu^{2}$ be the largest eigenvalue of $B^{T} B$ and $\sigma \neq \mu$ be the smallest eigenvalue of $B_{S}$. If there is a unit vector $u$ such that

$$
\sigma u=B_{S} u
$$




$$
\mu^{2} u=B^{T} B u,
$$

then there is a unit vector $v$ orthogonal to $u$ such that the span $S$ of $\{u, v\}$ is an invariant subspace of $B$ and the matrix of $B$ relative to $\{u, v\}$ is given by

$$
M=\left(\begin{array}{cc}
\sigma & -\sqrt{\mu^{2}-\sigma^{2}} \\
\sqrt{\mu^{2}-\sigma^{2}} & \sigma
\end{array}\right) .
$$

Proof. First, we show that

$$
\begin{gathered}
\left(B_{S} B_{A}-B_{A} B_{S}\right) u=0, \\
B_{A}^{2} u=\left(\sigma^{2}-\mu^{2}\right) u .
\end{gathered}
$$

We repeatedly use the fact that $B_{A}$ antisymmetric implies that $\left(B_{A} x, x\right)=0$. We have

$$
\begin{aligned}
\mu^{2} u & =B^{T} B u=\left(B_{S}-B_{A}\right)\left(B_{S}+B_{A}\right) u \\
& =\left(B_{S}^{2}-B_{A}^{2}\right) u+\left(B_{S} B_{A}-B_{A} B_{S}\right) u \\
& =\left(\sigma^{2} I-B_{A}^{2}\right) u+\left(B_{S}-\sigma I\right) B_{A} u
\end{aligned}
$$

Since $\left(B_{A} u, \mu^{2} u\right)=0$

$$
\begin{aligned}
0 & =\left(B_{A} u,\left(\sigma^{2} I-B_{A}^{2}\right) u+\left(B_{S}-\sigma I\right) B_{A} u\right) \\
& =\left(B_{A} u,\left(B_{S}-\sigma I\right) B_{A} u\right)
\end{aligned}
$$

Since $\sigma$ is the smallest eigenvalue of $B_{S}$, the square root of $B_{S}-\sigma I$ exists. Thus

$$
0=\left(\sqrt{B_{S}-\sigma I}\left(B_{A} u\right), \sqrt{B_{S}-\sigma I}\left(B_{A} u\right)\right),
$$

so $\left(B_{S}-\sigma I\right) B_{A} u=0$. This yields both of the claimed identities.

Now let $\alpha=\left\|B_{A} u\right\|=\sqrt{\left(B_{A} u, B_{A} u\right)}=\sqrt{\mu^{2}-\sigma^{2}}$ and $v=(1 / \alpha) B_{A} u$. Then $(v, u)=\left(B_{A} u, u\right)=0$. In addition,

$$
B_{S} v=\frac{1}{\alpha} B_{S} B_{A} u=\frac{1}{\alpha} B_{A} B_{S} u=\sigma v
$$

while

$$
B_{A} v=\frac{1}{\alpha} B_{A}^{2} u=\frac{\sigma^{2}-\mu^{2}}{\alpha} u=-\alpha u \text {. }
$$

This proves the lemma.

THEOREM 6.3. If $G$ is connected and not complete or a directed 3-cycle,

$$
D(G) \leq\left\lceil\frac{\ln (n-1)}{\ln \frac{\mu}{\sqrt{\mu^{2}-\sigma^{2}}}}\right\rceil
$$

where $\sigma$ is the smallest nonzero eigenvalue of $Q_{S}$ and $\mu=\|Q\|$.

Proof. Note that for a complete graph, $\mu=\sigma$, and for the directed 3-cycle, $\mu=\sqrt{3}$, while $\sigma=\frac{3}{2}$. Thus the inequality fails for these cases.

For any $\lambda$, we have

$$
\left\|I-\lambda Q-\frac{J}{n}\right\|^{2}=\max _{w \neq 0} \frac{\left(\left(I-\lambda Q-\frac{J}{n}\right) w,\left(I-\lambda Q-\frac{J}{n}\right) w\right)}{(w, w)} .
$$


Let $w^{\perp}=w-\left(w, u_{1}\right) u_{1}$, where $u_{1}=(1 / \sqrt{n})(1,1, \ldots, 1)^{*}$. Then $\left(w^{\perp}, u_{1}\right)=0$. Since $(I-\lambda Q-(J / n)) u_{1}=0$ and $(J / n) w^{\perp}=0$, we have

$$
\begin{aligned}
\frac{\left(\left(I-\lambda Q-\frac{J}{n}\right) w,\left(I-\lambda Q-\frac{J}{n}\right) w\right)}{(w, w)} & =\frac{\left((I-\lambda Q) w^{\perp},(I-\lambda Q) w^{\perp}\right)}{\left(w^{\perp}, w^{\perp}\right)+\left(w, u_{1}\right)^{2}} \\
& \leq \frac{\left(\left(I-2 \lambda Q_{S}+\lambda^{2} Q^{T} Q\right) w^{\perp}, w^{\perp}\right)}{\left(w^{\perp}, w^{\perp}\right)} \\
& \leq 1-2 \lambda \sigma+\lambda^{2} \mu^{2} .
\end{aligned}
$$

Setting $\lambda=\sigma / \mu^{2}$ yields

$$
\left\|I-\lambda Q-\frac{J}{n}\right\|^{2} \leq 1-\frac{\sigma^{2}}{\mu^{2}}<1
$$

since $G$ is connected. Thus

$$
\left\|I-\lambda Q-\frac{J}{n}\right\|=\frac{\sqrt{\mu^{2}-\sigma^{2}}}{\mu}<1 .
$$

If this is a strict inequality, then the theorem follows from Theorem 6.1. Equality can hold only if there is a vector $u$ such that $Q_{S} u=\sigma u$ and $Q^{T} Q u=\mu^{2} u$. Let $C=I-\lambda Q$. Let

$$
m=\frac{\ln (n-1)}{\ln \frac{\mu}{\sqrt{\mu^{2}-\sigma^{2}}}} .
$$

If we examine the proofs of Theorems 6.1 and 3.2, we find that $D(G) \leq\lfloor m\rfloor+1$, and that $D(G)>\lceil m\rceil$ can hold only if $m$ is an integer and if there exists $r \neq s$ such that

$$
-\frac{1}{n}=\left(e_{r},\left(C^{k}-\frac{J}{n}\right) e_{s}\right)=\left(f_{r},\left(C^{k}-\frac{J}{n}\right) f_{s}\right)=\left(f_{r}, C^{k} f_{s}\right)
$$

for $1 \leq k \leq m$ and $\|C-(J / n)\|=\sqrt{\mu^{2}-\sigma^{2}} / \mu$. Thus we can take $u=f_{s} /\left\|f_{s}\right\|=$ $\sqrt{n /(n-1)} f_{s}$ and $B=\tilde{Q}=\left(Q\right.$ restricted to the space orthogonal to $\left.u_{1}\right)$ in Lemma 6.2 and $v=1 / \sqrt{\mu^{2}-\sigma^{2}} B_{A} u$. The action of $C$ on $S=\{u, v\}$ is then given by

$$
M=\left(\begin{array}{cc}
1-\lambda \sigma & \lambda \alpha \\
-\lambda \alpha & 1-\lambda \sigma
\end{array}\right)
$$

and the action of $C^{2}$ is given by

$$
M^{2}=\left(\begin{array}{cc}
(1-\lambda \sigma)^{2}-(\lambda \alpha)^{2} & 2 \lambda \alpha(1-\lambda \sigma) \\
-2 \lambda \alpha(1-\lambda \sigma) & (1-\lambda \sigma)^{2}-(\lambda \alpha)^{2}
\end{array}\right)
$$

This yields the system

$$
\begin{gathered}
\left(f_{r}, C u\right)=(1-\lambda \sigma)\left(f_{r}, u\right)-\lambda \alpha\left(f_{r}, v\right) \\
\left(f_{r}, C^{2} u\right)=\left[(1-\lambda \sigma)^{2}-(\lambda \alpha)^{2}\right]\left(f_{r}, u\right)-2 \lambda \alpha(1-\lambda \sigma)\left(f_{r}, v\right)
\end{gathered}
$$


If we multiply these equations by $\sqrt{(n-1) / n}$, we obtain

$$
\begin{gathered}
\left(f_{r}, C f_{s}\right)=(1-\lambda \sigma)\left(f_{r}, f_{s}\right)-\lambda \alpha \sqrt{\frac{n-1}{n}}\left(f_{r}, v\right) \\
\left(f_{r}, C^{2} f_{s}\right)=\left[(1-\lambda \sigma)^{2}-(\lambda \alpha)^{2}\right]\left(f_{r}, f_{s}\right)-2 \lambda \alpha(1-\lambda \sigma) \sqrt{\frac{n-1}{n}}\left(f_{r}, v\right) .
\end{gathered}
$$

If $m \geq 2$ and we use $\left(f_{r}, f_{s}\right)=-1 / n$, we obtain

$$
\begin{gathered}
-\frac{1}{n}=-\frac{1}{n}(1-\lambda \sigma)-\lambda \alpha \sqrt{\frac{n-1}{n}}\left(f_{r}, v\right), \\
-\frac{1}{n}=-\frac{1}{n}\left[(1-\lambda \sigma)^{2}-(\lambda \alpha)^{2}\right]-2 \lambda \alpha(1-\lambda \sigma) \sqrt{\frac{n-1}{n}}\left(f_{r}, v\right) .
\end{gathered}
$$

Elimination yields

$$
\frac{\sigma^{2}}{\mu^{4}}\left(\mu^{2}-\sigma^{2}\right)=(\lambda \alpha)^{2}=0,
$$

which has no roots but $\sigma=0$ or $\mu^{2}=\sigma^{2}$.

Thus the only possibilities are $\sigma=\mu$ or $m=1$. If $\sigma=\mu$, then all the eigenvalues of $B_{S}$ and all the singular values of $B$ are equal to $\mu$. Thus every vector $u$ satisfies the conditions of Lemma 6.2. In particular, $B_{A}^{2}=0$, so $B=B_{S}=\mu I$ and $Q=$ $\mu(I-(J / n))$. Since the entries of $Q$ must be integers, $\mu=n$. Thus the graph is the complete graph.

If $m=1$, then $\mu^{2} /\left(\mu^{2}-\sigma^{2}\right)=(n-1)^{2}$, and so $\|I-\lambda Q-(J / n)\|=1 /(n-1)$. Because of $\left(f_{r},(I-\lambda Q-(J / n)) f_{s}\right)=-1 / n$, we have, in this case,

$$
\left(I-\lambda Q-\frac{J}{n}\right) f_{s}=-\frac{1}{n-1} f_{r}
$$

This leads to

$$
\frac{\sigma}{\mu^{2}} Q e_{s}=\lambda Q e_{s}=e_{s}+\frac{1}{n-1} e_{r}-\frac{1}{n-1} w
$$

and so

$$
\left(e_{s}, Q e_{s}\right)=\frac{\mu^{2}}{\sigma} \frac{n-2}{n-1}
$$

If $n>2$, then there is a $t \neq r, s$, and

$$
\left(e_{t}, Q e_{s}\right)=-\frac{\mu^{2}}{\sigma} \frac{1}{n-1} .
$$

Since the off-diagonal entries of $Q$ are 0 or -1 , we must have $\mu^{2} / \sigma=n-1$. Thus $\left(e_{s}, Q e_{s}\right)=n-2$. We may also solve for both $\mu$ and $\sigma$ to obtain $\mu=\sqrt{n(n-2)}$ and $\sigma=n(n-2) /(n-1)$. Let prime denote parameters relative to the complement of $G$. We have

$$
Q_{S}^{\prime}+Q_{S}=n\left(I-\frac{J}{n}\right)
$$

Also, note that

$$
\sigma_{\max }\left(Q_{S}^{\prime}\right)=\max _{\|x\|=1}(Q x, x) \geq \max _{i}\left(e_{i}, Q e_{i}\right)=\max _{i} d_{i}\left(G^{\prime}\right)
$$


Thus

$$
\frac{n(n-2)}{n-1}=\sigma=n-\sigma_{\max }\left(Q_{S}^{\prime}\right) \leq n-\max _{i} d_{i}\left(Q_{S}^{\prime}\right)
$$

so if $n>2$,

$$
\max _{i} d_{i}\left(Q_{S}^{\prime}\right) \leq 1+\frac{1}{n-1}<2 .
$$

Thus each vertex of $G^{\prime}$ has degree 0 or 1 , so $G^{\prime}$ is the disjoint union of isolated vertices and directed cycles $D_{k_{i}}$ with $\sum k_{i} \leq n$. We need

$$
\begin{aligned}
1+\frac{1}{n-1} & =n-\sigma=\sigma_{\max }\left(Q_{S}^{\prime}\right)=\max _{i} \sigma_{\max }\left(Q\left(D_{K_{i}}\right)\right)_{S} \\
& =\frac{1}{2} \max _{i} \sigma_{\max }\left(Q\left(C_{k_{i}}\right)\right) \\
& = \begin{cases}2 & \text { if some } k_{i} \text { is even, } \\
1-\cos \frac{2 \pi}{k}, k=\max _{i} k_{i} & \text { if no } k_{i} \text { is even. }\end{cases}
\end{aligned}
$$

However, we can never have $-\cos (2 \pi / k)=1 /(n-1)$ unless $k=3$ and $n=3$; that is, $G$ is a directed 3-cycle. This contradiction eliminates this case and completes the proof of the theorem.

7. Normal matrices. If we compare the result of Theorem 6.3 with those obtained from Theorem 5.3 for undirected graphs, we see a marked difference. We would like to extend the method of $\S 5$ to directed graphs. We can do this fairly easily for one class of directed graphs, namely, those whose adjacency matrix is normal. A matrix $A$ is normal if $A A^{*}=A^{*} A$. We have the following well-known fact. (For the application of normal matrices to iterative methods, see [5], which also includes some other important properties of normal matrices.)

LEMMA 7.1. The following properties of a matrix $A$ are equivalent:

(i) $A^{*} A=A A^{*}$,

(ii) $A^{*}$ is a polynomial in $A$,

(iii) the eigenvectors of $A$ form a complete orthonormal basis.

THEOREM 7.2. Suppose that a directed graph with $n$ vertices has Laplacian $Q$ such that $Q Q^{T}=Q^{T} Q$ and that all the eigenvalues of $Q$ except 0 are contained in an ellipse with center $\delta>0$, foci $\delta \pm \gamma, \gamma>0$, and semi-major axis of length $\alpha$, where $\gamma<\alpha<\delta$. If $m$ is the least integer such that

$$
\frac{T_{m}\left(\frac{\alpha}{\gamma}\right)}{T_{m}\left(\frac{\alpha}{\delta}\right)}<\frac{1}{n-1},
$$

then $D(G) \leq m$. Furthermore, suppose that all of the eigenvalues of $Q$ except 0 are contained in an ellipse with center $\delta>0$, foci $\delta \pm i \gamma, \gamma>0$, and semi-major axis of length $\alpha, \gamma<\alpha<\sqrt{\delta^{2}+\gamma^{2}}$. If $m$ is the least even integer such that

$$
\frac{T_{m}\left(\frac{\alpha}{\gamma}\right)}{T_{m}\left(\sqrt{1+\left(\frac{\delta}{\gamma}\right)^{2}}\right)}<\frac{1}{n-1}
$$

then $D(G) \leq m$. 
Proof. The proof relies on the remarks of $\S 4$ and is similar to the proof of Theorem 5.3. We leave it to the reader.

The following lemma may be of some use in estimating the diameter of directed graphs in conjunction with Theorem 7.2.

LEMMA 7.3. Let $Q$ be the Laplacian of a connected directed graph with in-degree equal to out-degree. Then the eigenvalues of $Q$ lie in a disk of radius $\Delta=\max \{d(u) \mid u \in$ $G\}$ with center at $\Delta$. There is exactly one zero eigenvalue. Furthermore, if there are $k>1$ eigenvalues on the boundary of the disk $|z-\Delta|=\Delta$, then these eigenvalues have the form $\left(1-e^{(2 \pi m / k) i}\right) \Delta$, and $G$ is $k$-partite.

Proof. That the eigenvalues $\lambda$ satisfy $|\lambda-\Delta| \leq \Delta$ follows from the Gershgorin theorem. Now consider the nonnegative matrix $B=\Delta I-Q$. The Perron-Frobenius theorem yields the result.

8. Estimating the ellipse which encloses the eigenvalues of a real normal matrix. It is easy to recognize when Theorem 7.2 applies. For example, if $\Gamma$ is a group and $\Delta=\left\{\delta_{1}, \delta_{2}, \ldots, \delta_{d}\right\}$ are generators, the Cayley graph $G(\Gamma, \Delta)$ has a normal adjacency matrix if and only if, for each $g \in \Gamma$, the number of solutions $\left(\delta_{i}, \delta_{j}\right)$ to $\delta_{i} g=\delta_{j}$ is the same as the number of solutions $\left(\delta_{i}, \delta_{j}\right)$ to $g \delta_{i}=\delta_{j}$. Thus, if $\Gamma$ is abelian, $Q$ is normal. We need a method for finding the ellipse in Theorem 7.2.

Suppose that the eigenvalues of $Q$ are known. There are an infinite number of ellipses symmetric with respect to the real axis that enclose the nonzero eigenvalues of $Q$ and exclude zero. We would like to choose the ellipse that minimizes $m$ in Theorem 7.2. Equivalently, given any $m$, we would like to find the ellipse that minimizes the bounds in Theorem 7.2. To our knowledge, this problem is unsolved. However, a related problem has been solved and may be useful. Using the definition $T_{m}(z)=$ $\cosh \left(n \cosh ^{-1}(z)\right)$ and making use of the formula $\cosh ^{-1}(z)=\ln \left(z+\left(z^{2}-1\right)^{1 / 2}\right)$, we may write $g(z)=z+\sqrt{z^{2}-1}, f(z)=z+\sqrt{z^{2}+1}$, and

$$
\frac{T_{m}\left(\frac{\alpha}{\gamma}\right)}{T_{m}\left(\frac{\delta}{\gamma}\right)}=\left(\frac{\alpha+\sqrt{\alpha^{2}-\gamma^{2}}}{\delta+\sqrt{\delta^{2}+\gamma^{2}}}\right)^{m}\left(\frac{1+g\left(\frac{\alpha}{\gamma}\right)^{-2 m}}{1+g\left(\frac{\delta}{\gamma}\right)^{-2 m}}\right)
$$

for the case of real foci, and

$$
\frac{T_{m}\left(\frac{\alpha}{\gamma}\right)}{T_{m}\left(\frac{\sqrt{\delta^{2}+\gamma^{2}}}{\gamma}\right)}=\left(\frac{\alpha+\sqrt{\alpha^{2}-\gamma^{2}}}{\delta+\sqrt{\delta^{2}+\gamma^{2}}}\right)^{m}\left(\frac{1+g\left(\frac{\alpha}{\gamma}\right)^{-2 m}}{1+f\left(\frac{\delta}{\gamma}\right)^{-2 m}}\right)
$$

for complex foci and $m$ even.

The second term in each expression is bounded by 2 and rapidly approaches $L$ for large $m$. The problem of finding the ellipse symmetric with respect to the real axis that encloses a given set of points and excludes zero that minimizes the first term in the above expression is solved in Manteuffel [10]. The solution depends only on the convex hull of the nonzero spectrum of $Q$. If the best such ellipse has real foci, then the smallest $m$ that satisfies

$$
\frac{T_{m}\left(\frac{\alpha}{\gamma}\right)}{T_{m}\left(\frac{\delta}{\gamma}\right)}<\frac{1}{n-1}
$$


can be found. If the best such ellipse has complex foci, then the smallest even $m$ that satisfies

$$
\frac{T_{m}\left(\frac{\alpha}{\gamma}\right)}{T_{m}\left(\frac{\left(\delta^{2}+\gamma^{2}\right)^{1 / 2}}{\gamma}\right)}<\frac{1}{n-1}
$$

can be found. Application of Theorem 7.2 then yields the bound $D(G) \leq m$.

9. Estimating the spectrum of $Q$. The theorems in the above sections require knowledge of the extremal eigenvalues of $Q$. If the eigenvalues of $Q$ are not known, they can be computed by a variety of methods. If the dimension of $Q$ is not too large, good algorithms exist for computing the entire spectrum. If the dimension is large, it may be more practical to estimate the extremal eigenvalues by iterative methods. If systems of the type $(\lambda I-Q) x=y$ are easily solved, then inverse iteration may be employed (cf. Wilkinson [17]). If not, then methods based upon taking orthogonal sections of $Q$ may be used, for example, the Lanczos algorithm (cf. [8]). These methods only require repeated matrix vector multiplication.

Suppose that $Q$ is symmetric. The Lanczos algorithm requires an initial vector, say $r$, and iteratively forms a tridiagonal matrix $T_{k}$ that is the orthogonal section of $Q$ onto the Krylov subspace $K_{k}(r, Q)=s p\left\{r, Q r, \ldots, Q^{k-1} r\right\}$. The eigenvalues of $T_{k}$ yield good approximations to the extremal eigenvalues of $Q$. If $r$ is chosen to be orthogonal to the null vector $u_{1}$, then the extremal eigenvalues of $T_{k}$ approximate $\lambda_{2}$ and $\lambda_{n}$. Estimates of the rate of convergence are available [4].

If $Q$ is nonsymmetric then Arnoldi's method [15], [2] yields an upper Hessenberg matrix $H_{k}$ instead of $T_{k}$ as the orthogonal section of $Q$ onto the Krylov space $K_{k}(r, Q)$. The eigenvalues of $H_{k}$ again yield approximations to the extremal eigenvalues of $Q$. In this case, however, the convex hull of the spectrum of $H_{k}$ approximates the convex hull of the spectrum of $Q$. Again, if $r$ is chosen orthogonal to $u_{1}$, the approximations ignore the zero eigenvalue. If $Q$ is normal, the only case in which we seek the spectrum of a nonsymmetric $Q$, the procedure yields good approximations. The adaptive methods described in [11] simultaneously find the eigenvalues and the optimal ellipse.

For general $Q$, we are limited to Theorem 6.2. This requires $\mu=\|Q\|$, which can be computed by applying the Lanczos procedure on $Q^{T} Q$ to find $\mu^{2}=\left\|Q^{T} Q\right\|$. The value $\sigma=\lambda_{2}\left(Q_{S}\right)$ can also be approximated by the Lanczos procedure applied to $Q_{S}$.

\section{REFERENCES}

[1] N. Alon ANd V. D. MlLman, $\lambda_{1}$, Isoperimetric inequalities for graphs and superconcentrators, J. Combin. Theory Ser. B, 38 (1985), pp. 73-88.

[2] W. E. ARNOLDI, The principal of minimized iteration in the solution of the matrix eigenvalue problem, Quart. Appl. Math., 9 (1951), pp. 17-29.

[3] F. R. K. Chung, Diameters and eigenvalues, J. Amer. Math. Soc., 2 (1989), pp. 187-196.

[4] J. J. Dongarra, J. R. Bunch, C. B. Moler, And G. W. Stewart, LiNPACK User's Guide, Society for Industrial and Applied Mathematics, Philadelphia, PA, 1979.

[5] V. FABER AND T. A. MANTEUFFEL, Necessary and sufficient conditions for the existence of a conjugate gradient method, SIAM J. Numer. Anal., 21 (1984), pp. 352-362.

[6] B. Fischer AND P. FREUND, On the constrained Chebychev approximation problem on ellipses, J. Approx. Theory, 62 (1990), pp. 297-315.

[7] - Chebychev polynomials are not always optimal, J. Approx. Theory, 65 (1991), pp. $261-272$.

[8] G. A. Golub And C. F. VAn LoAn, Matrix Computations, Johns Hopkins Univ. Press, Baltimore, MD, 1983. 
[9] A. Lubotzky, R. Phillips, and P. Sarnak, Ramanujan graphs, Combinatorica, 8 (1988), pp. $261-277$.

[10] T. A. Manteuffel, The Tchebychev iteration for nonsymmetric systems, Numer. Math., 28 (1977), pp. 307-327.

[11] - The adaptive procedure for estimating parameters for the nonsymmetric Tchebychev iteration, Numer. Math., 31 (1978), pp. 183-208.

[12] B. MoHAR, Eigenvalues, diameter, and mean distance in graphs, Graphs Combin., 7 (1991), pp. 53-64.

[13] - The Laplacian spectrum of graphs, in Graph Theory, Combinatorics and Applications 2 (Kalamazoo, MI, 1988), Wiley-Intersciences, pp. 871-898.

[14] T. J. Rivlin, The Chebyshev Polynomials, John Wiley, New York, 1990.

[15] Y. SAAD, Variations on Arnoldi's method for computing eigenelements of large unsymmetric matrices, Linear Algebra Appl., 34 (1980), pp. 269-295.

[16] P. SARnaK, Some Applications of Modular Forms, Cambridge University Press, Cambridge, UK, 1990.

[17] J. H. Wilkinson, Algebraic Eigenvalue Problem, Clarendon Press, Oxford, UK, 1965. 